Pacific Journal of Mathematic 


\title{
ON PARTIAL ISOMETRIES WITH NO ISOMETRIC PART
}

\section{JAMES GUYKER}

\begin{abstract}
P. R. Halmos and L. J. Wallen characterized partial isometries all of whose positive integral powers are partial isometries on Hilbert space as unique direct sums of unitary operators, pure isometries, pure co-isometries and truncated shifts, with each type of summand occurring at most once. In the present paper, this structure is extended to partial isometries $T$ with no isometric part whose first $N+1$ powers are partial isometries. Moreover, if $T=\Sigma \oplus T_{1} \oplus V$, it is shown that every projection $P$ commuting with $T$ is of the form $P=\Sigma \oplus P, \oplus Q$ where $P$, and $Q$ are projections commuting with $T$, and $V$ respectively. The canonical model of $L$. de Branges and $J$. Rovnyak is used to explicitly describe the structure of the reducing subspaces of $T$ in terms of the characteristic operator-function of $T^{*}$, from which this result follows. A direct proof is also obtained using a general reducing subspace structure theorem for arbitrary contractions with no isometric part.
\end{abstract}

1. Preliminaries. Let $\mathscr{C}$ be a complex, separable Hilbert space. The space $\mathscr{H}^{2}(\mathscr{C})$ is the Hilbert space of analytic, squareintegrable functions $f(z)$ defined on the open unit disk with values in $\mathscr{C}$ such that the set

$$
I=\left\{\frac{1}{2 \pi} \int_{0}^{2 \pi}\left|f\left(r e^{i \theta}\right)\right|^{2} d \theta: 0<r<1\right\}
$$

where $|\cdot|$ is the norm in $\mathscr{C}$, is bounded, with the norm given by $\|f(z)\|^{2}=\sup I$. If $f(z)$ is in $\mathscr{H}^{2}(\mathscr{C})$, then there is a square-summable sequence $\left\{a_{n}: n=0,1,2, \cdots\right\}$ of vectors in $\mathscr{C}$ such that for each $|z|<1$, $f(z)=\Sigma a_{n} z^{n}$ in the metric of $\mathscr{C}$, and moreover $\|f(z)\|^{2}=\Sigma\left|a_{n}\right|^{2}$.

A function $B(z)$ defined on the open unit disk whose values are operators on $\mathscr{C}$ will be called analytic if there is a sequence $\left\{B_{n}: n=0,1,2, \cdots\right\}$ of operators on $\mathscr{C}$ such that for every $|z|<1$, $B(z)=\Sigma B_{n} z^{n}$, where the series converges in the strong operator topology for each fixed $z$. An analytic function $B(z)$ is bounded if there exists a constant $M$ such that $|B(w)| \leqq M$ for every $|w|<1$. If $B(z)$ is analytic and bounded, and $B(z)=\Sigma B_{n} z^{n}$, then for every $f(z)=\Sigma a_{n} z^{n}$ in $\mathscr{H}^{2}(\mathscr{C})$, the Cauchy product $B(z) f(z)=\Sigma\left(\sum_{k=0}^{n} B_{k} a_{n-k}\right) z^{n}$ is in $\mathscr{H}^{2}(\mathscr{C})$ and defines a bounded operator, which will be denoted by $T_{B}$, on $\mathscr{H}^{2}(\mathscr{C})$. Finally, we 
recall that a bounded, analytic function $B(z)$ is an inner function if $T_{B}$ is a partial isometry on $\mathscr{H}^{2}(\mathscr{C})$.

If $E$ is a subset of a Hilbert space $\mathscr{H}$, then $C L(E)$ and $\vee E$ will denote the closure and closed span of $E$ respectively. For subspaces $M$ and $N$ of $\mathscr{H}$, if $M \perp N$, then $M \oplus N$ will denote the orthogonal direct sum of $M$ and $N$; if $N \subset M$, then $M \ominus N$ will be the orthogonal complement of $N$ in $M$.

The proofs of the following results may be found in [1] or [2].

Let $B(z)$ be an analytic function that is bounded by $M=1$. The de Branges-Rovnyak space $\mathscr{H}(B)$ is the Hilbert space of functions $f(z)$ in $\mathscr{H}^{2}(\mathscr{C})$ such that the set

$$
J=\left\{\|f(z)+B(z) g(z)\|^{2}-\|g(z)\|^{2}: g(z) \in \mathscr{H}^{2}(\mathscr{C})\right\}
$$

is bounded, with the norm given by $\|f(z)\|_{B}^{2}=\sup J$. Since the zero-function is in $\mathscr{H}^{2}(\mathscr{C})$, it follows that $\|f(z)\|_{B} \geqq\|f(z)\|$ for every $f(z)$ in $\mathscr{H}(B)$, so that $\mathscr{H}(B)$ is continuously embedded in $\mathscr{H}^{2}(\mathscr{C})$. The space $\mathscr{H}(B)$ is isometrically embedded in $\mathscr{H}^{2}(\mathscr{C})$ if and only if $B(z)$ is inner, in which case $\mathscr{H}(B) \oplus\left(\right.$ range $\left.T_{B}\right)=\mathscr{H}^{2}(\mathscr{C})$. For a subset $E$ of $\mathscr{H}(B)$, the notation $C L_{B}(E)$ and $\vee_{B} E$ will be used to emphasize that the corresponding operations are taken in the metric of $\mathscr{H}(B)$.

The function

$$
K(w, z)=\frac{1-B(z) \bar{B}(w)}{1-z \bar{w}}
$$

where for each $|w|<1, \bar{B}(w)$ is the adjoint of $B(w)$ on $\mathscr{C}$, is a reproducing kernel function for $\mathscr{H}(B)$ : for each vector $c$ in $\mathscr{C}$ and for each $|w|<1$, $K(w, z) c$ is in $\mathscr{H}(B)$, and if $f(z)$ is any element of $\mathscr{H}(B)$, then

$$
\langle f(w), c\rangle=\langle f(z), K(w, z) c\rangle_{B} .
$$

If $f(z)$ is in $\mathscr{H}(B)$, then $(f(z)-f(0)) / z$ is in $\mathscr{H}(B)$ and

$$
\left\|\frac{f(z)-f(0)}{z}\right\|_{B}^{2} \leqq\|f(z)\|_{B}^{2}-|f(0)|^{2}
$$

A contraction $T$ on a Hilbert space $\mathscr{H}$ is said to have no isometric part if there is no nonzero vector $f$ in $\mathscr{H}$ such that $\left\|T^{n} f\right\|=\|f\|$ for every $n=1,2, \cdots$. Let $T$ be an arbitrary contraction with no isometric part. Then $T$ is unitarily equivalent to the backward shift operator

$$
R(0): f(z) \rightarrow \frac{f(z)-f(0)}{z}
$$


acting on a space $\mathscr{H}(B)$, for some coefficient space $\mathscr{C}$ and some analytic function $B(z)$ which is bounded by 1 . Furthermore, the difference quotient identity holds in the space $\mathscr{H}(B):\|R(0) f(z)\|_{B}^{2}=\|f(z)\|_{B}^{2}-|f(0)|^{2}$ for every $f(z)$ in $\mathscr{H}(B)$. The function $B(z)$ is called the characteristic operator-function of $T^{*}$.

The operator $R(0)^{*}$ on $\mathscr{H}(B)$ is related to $R(0)$ on $\mathscr{H}\left(B^{*}\right)$ where $B^{*}(z)=\Sigma \bar{B}_{n} z^{n}$ if $B(z)=\sum B_{n} z^{n}$ and $\bar{B}_{n}$ is the adjoint of $B_{n}$ on $\mathscr{C}$. Associated with the space $\mathscr{H}(B)$ is a space which is useful in studying $R(0)$ on $\mathscr{H}(B)$ when the difference quotient identity fails to hold in $\mathscr{H}(B)$ : the space $\mathscr{D}(B)$ is the Hilbert space of pairs $(f(z), g(z))$ in $\mathscr{H}(B) \times \mathscr{H}\left(B^{*}\right)$ such that if $g(z)=\sum a_{n} z^{n}$ then $h_{n}(z)=z^{n} f(z)-B(z) \times$ $\left(a_{0} z^{n-1}+a_{1} z^{n-2}+\cdots+a_{n-1}\right)$ belongs to $\mathscr{H}(B)$ for every $n=1,2, \cdots$, and the set

$$
K=\left\{\left\|h_{n}(z)\right\|_{B}^{2}+\left|a_{0}\right|^{2}+\left|a_{1}\right|^{2}+\cdots+\left|a_{n-1}\right|^{2}: n=1,2, \cdots\right\}
$$

is bounded, with the norm given by $\|(f(z), g(z))\|_{\mathscr{D}}^{2}=\sup K$.

If $(f(z), g(z))$ is in $\mathscr{D}(B)$, then

$$
(u(z), v(z))=\left(R(0) f(z), z g(z)-B^{*}(z) f(0)\right)
$$

is in $\mathscr{D}(B)$ and $\|(u(z), v(z))\|_{\mathscr{D}}^{2}=\|(f(z), g(z))\|_{\mathscr{D}}^{2}-|f(0)|^{2}$. The difference quotient operator on $\mathscr{D}(B)$ is the bounded linear transformation

$$
D:(f(z), g(z)) \rightarrow(u(z), v(z))
$$

whose adjoint is given by $D^{*}(f(z), g(z))=(z f(z)-B(z) g(0), R(0) g(z))$ and satisfies $\left\|D^{*}(f(z), g(z))\right\|_{\mathscr{D}}^{2}=\|(f(z), g(z))\|_{\mathscr{D}}^{2}-|g(0)|^{2}$. for every $(f(z), g(z))$ in $\mathscr{D}(B)$.

2. Reducing Subspaces of $R(0)$. We recall that a (closed) subspace $M$ of a Hilbert space $\mathscr{H}$ reduces a bounded operator $T$ on $\mathscr{H}$ if $M$ is invariant under both $T$ and $T^{*}$. The structure of the reducing subspaces of the backward shift on $\mathscr{H}(B)$ whenever $B(z)$ is a constant operator-function is well-known [3, Lemma 3.2], [9, Theorem 1]: if $B(z)=B(0)$ and $|B(0)| \leqq 1$, then the only reducing subspaces of $R(0)$ on $\mathscr{H}(B)=\vee_{B}\left\{R(0)^{* n} \mathscr{K}: n=0,1,2, \cdots\right\}$, where $\mathscr{K}$ is the kernel of $R(0)$, are the slices $M=\vee_{B}\left\{R(0)^{* n} S: n=0,1,2, \cdots\right\}$ where $S$ is an arbitrary subspace of $\mathscr{K}$. Furthermore, $\mathscr{H}(B) \ominus M=\mathrm{\vee}_{B}\left\{R(0)^{* n}(\mathscr{K} \Theta S): \quad n=\right.$ $0,1,2, \cdots\}$. In this section, which is a continuation of [6] and [7], it is shown that this structure is essentially preserved under general conditions on $B(z)$. We first establish two auxiliary results.

Lemma 2.1. Let $\mathscr{H}(B)$ in $\mathscr{H}^{2}(\mathscr{C})$ be a de Branges-Rovnyak space 
with reproducing kernel function $K(w, z)$. Then $\mathscr{H}(B)=$ $\vee_{B}\left\{R(0)^{* n} K(0, z) \mathscr{C}: n=0,1,2, \cdots\right\}$ and the following are equivalent for $a$ subspace $M$ of $\mathscr{H}(B)$.

(1) $\quad M=\vee_{B}\left\{R(0)^{* n} K(0, z) S: n=0,1,2, \cdots\right\}$ for some subspace $S$ of $\mathscr{C}$ which is invariant under $A_{i j}=B_{i} \bar{B}_{j}$ for all $i, j=0,1,2, \cdots$.

(2) $\quad M=\mathscr{H}(B) \cap N$ for some subspace $N$ of $\mathscr{H}^{2}(\mathscr{C})$ which reduces both $T_{z}$ and $1-T_{B} T_{B}^{*}$ on $\mathscr{H}^{2}(\mathscr{C})$.

In this case,

$$
\begin{aligned}
\mathscr{H}(B) \ominus M & =\bigvee_{B}\left\{R(0)^{* n} K(0, z)(\mathscr{C} \ominus S): n=0,1,2, \cdots\right\} \\
& =\mathscr{H}(B) \cap\left(\mathscr{H}^{2}(\mathscr{C}) \ominus N\right) .
\end{aligned}
$$

Proof. We first observe that $\mathscr{H}(B) \cap N=C L_{B}\left(1-T_{B} T_{B}^{*}\right) N$ for any reducing subspace $N$ of $1-T_{B} T_{B}^{*}$. This follows since $1-T_{B} T_{B}^{*}$ is continuous as an operator from $\mathscr{H}^{2}(\mathscr{C})$ into $\mathscr{H}(B)$, and $\langle f(z),(1-$ $\left.\left.T_{B} T_{B}^{*}\right) f(z)\right\rangle_{B}=\|f(z)\|^{2}$ for every $f(z)$ in $\mathscr{H}(B)[1$, Theorem 4]. Also note that

$$
\begin{aligned}
\mathscr{H}(B) & =C L_{B}\left(1-T_{B} T_{B}^{*}\right) \mathscr{H}(B)=C L_{B}\left(1-T_{B} T_{B}^{*}\right) \mathscr{H}^{2}(\mathscr{C}) \\
& =C L_{B}\left(1-T_{B} T_{B}^{*}\right) N \oplus C L_{B}\left(1-T_{B} T_{B}^{*}\right)\left(\mathscr{H}^{2}(\mathscr{C}) \ominus N\right)
\end{aligned}
$$

since for any $f(z)$ in $N$ and $g(z)$ in $\mathscr{H}^{2}(\mathscr{C}) \ominus N$,

$$
\left\langle\left(1-T_{B} T_{B}^{*}\right) f(z),\left(1-T_{B} T_{B}^{*}\right) g(z)\right\rangle_{B}=\left\langle\left(1-T_{B} T_{B}^{*}\right) f(z), g(z)\right\rangle=0 .
$$

(1) implies (2). Suppose that $M=\vee_{B}\left\{R(0)^{* n} K(0, z) S: \quad n=\right.$ $0,1,2, \cdots\}$ for some subspace $S$ of $\mathscr{C}$ such that $B_{i} \bar{B}, S \subseteq S$ for all $i, j=0,1,2, \cdots$. Let $N=\vee\left\{T_{z}^{n} S: n=0,1,2, \cdots\right\}$. Clearly $N$ reduces $T_{z}$ on $\mathscr{H}^{2}(\mathscr{C})$. Moreover, since $\left(1-T_{B} T_{B}^{*}\right) S=K(0, z) S$, and for $n \geqq 1$, $\left(1-T_{B} T_{B}^{*}\right) T_{z}^{n} S=z\left(1-T_{B} T_{B}^{*}\right) T_{z}^{n-1} S-B(z) \bar{B}_{n} S$, it follows that $N$ reduces $1-T_{B} T_{B}^{*}$ on $\mathscr{H}^{2}(\mathscr{C})$. Hence by the above remarks,

$$
\begin{aligned}
\mathscr{H}(B) \cap N & =C L_{B}\left(1-T_{B} T_{B}^{*}\right) N \\
& =\bigvee_{B}\left\{\left(1-T_{B} T_{B}^{*}\right) T_{z}^{n} S: n=0,1,2, \cdots\right\} \\
& =\bigvee_{B}\left\{R(0)^{* n} K(0, z) S: n=0,1,2, \cdots\right\}=M .
\end{aligned}
$$

Similarly, since $\mathscr{H}^{2}(\mathscr{C}) \ominus N=\vee\left\{T_{z}^{n}(\mathscr{C} \ominus S): n=0,1,2, \cdots\right\}$, we have that 


$$
\begin{aligned}
\mathscr{H}(B) \ominus M & =\mathscr{H}(B) \ominus C L_{B}\left(1-T_{B} T_{B}^{*}\right) N \\
& =C L_{B}\left(1-T_{B} T_{B}^{*}\right)\left(\mathscr{H}^{2}(\mathscr{C}) \ominus N\right) \\
& =\bigvee_{B}\left\{R(0)^{* n} K(0, z)(\mathscr{C} \ominus S): n=0,1,2, \cdots\right\} .
\end{aligned}
$$

(2) implies (1). Suppose that $M=\mathscr{H}(B) \cap N$ where $N$ is a subspace of $\mathscr{H}^{2}(\mathscr{C})$ with reduces both $T_{z}$ and $1-T_{B} T_{B}^{*}$ on $\mathscr{H}^{2}(\mathscr{C})$. There exists a unique subspace $S$ of $\mathscr{C}$ such that $N=\vee\left\{T_{z}^{n} S: n=0,1,2, \cdots\right\}$ and $\mathscr{H}^{2}(\mathscr{C}) \ominus N=\vee\left\{T_{z}^{n}(\mathscr{C} \ominus S): n=0,1,2, \cdots\right\}$. Therefore,

$$
\begin{aligned}
M & =C L_{B}\left(1-T_{B} T_{B}^{*}\right) N=\underset{B}{\vee}\left\{\left(1-T_{B} T_{B}^{*}\right) T_{z}^{n} S: n=0,1,2, \cdots\right\} \\
& =\underset{B}{\vee}\left\{R(0)^{* n} K(0, z) S: n=0,1,2, \cdots\right\}
\end{aligned}
$$

and similarly for $\mathscr{H}(B) \ominus M$. By the structure of $N$ and the above identities for $\left(1-T_{B} T_{B}^{*}\right) T_{z}^{n} S$, it follows that $S$ is invariant under $A_{i j}=B_{\imath} \bar{B}_{J}$ for all $i, j=0,1,2, \cdots$.

We recall that an operator $T$ on a Hilbert space $\mathscr{H}$ is a partial isometry if $\|T f\|=\|f\|$ for every vector $f$ in $\mathscr{H}$ which is orthogonal to the kernel of $T$ (or equivalently, if $T=T T^{*} T$ ) [8].

LEMMA 2.2. Let $D$ be the difference quotient operator on the de Branges-Rovnyak space $\mathscr{D}(B)$. Then $B_{0}, B_{1}, \cdots, B_{N}$ are partial isometries on the coefficient space $\mathscr{C}$ if and only if $D, D^{2}, \cdots, D^{N+1}$ are partial isometries on $\mathscr{D}(B)$, in which case $B_{\imath} \bar{B}_{\jmath}=\bar{B}_{i} B_{j}=0$ for all $i$ and $j$ such that $i \neq j$ and either $0 \leqq i \leqq N$ or $0 \leqq j \leqq N$.

Proof. For $f(z)$ in $\mathscr{H}^{2}(\mathscr{C})$ let $f_{n}(0)$ denote the coefficient of $z^{n}$ in the Taylor's series expansion of $f$. Then by the defining equations of $D$, we have for $(f(z), g(z))$ in $\mathscr{D}(B)$ and any positive integer $k$,

$$
\left(D^{k} D^{* k} D^{k}\right)(f(z), g(z))=D^{k}(f(z), g(z))-\left(F_{k}(z), G_{k}(z)\right)
$$

where for $j, k \geqq 1$,

$$
\begin{aligned}
g^{0}(z) & =g(z), g^{\prime}(z)=z g^{j-1}(z)-B^{*}(z) f_{j-1}(0) \\
f^{0}(z) & =R(0)^{k} f(z), f^{j}(z)=z f^{j-1}(z)-B(z) g_{\jmath^{-1}}^{k}(0), \\
\left(h^{0}(z)\right. & \left.=R(0)^{k} g^{k}(z), h^{j}(z)=z h^{\prime-1}(z)-B^{*}(z) f_{j-1}^{k}(0)\right) \\
F_{k}(z) & =\sum_{n=0}^{k-1} R(0)^{n} \frac{B(z)-B(0)}{z} g_{n}^{k}(0), \quad \text { and } \\
G_{k}(z) & =\sum_{n=0}^{k-1}\left(g_{n}^{k}(0)+B^{*}(z) f_{k-n-1}^{k}(0)\right) z^{n}
\end{aligned}
$$


Suppose that $B_{0}, B_{1}, \cdots, B_{N}$ are partial isometries on $\mathscr{C}$. For every vector $c$ in $\mathscr{C}$ which is orthogonal to the kernel of $B$, for fixed $j, 0 \leqq j \leqq N$, we have that

$$
|c|^{2} \geqq\|B(z) c\|^{2}=\left|B_{j} c\right|^{2}+\sum_{i \neq j}\left|B_{l} c\right|^{2}=|c|^{2}+\sum_{i \neq j}\left|B_{i} c\right|^{2}
$$

so that $B_{i} c=0$ for all $i \neq j$. Since $i$ and $j$ were arbitrarily chosen, it follows that $B_{i} \bar{B}_{j}=0$ for all $i$ and $j$ such that $i \neq j$ and $0 \leqq j \leqq N$. Since the operator-function $B^{*}(z)=\Sigma \bar{B}_{n} z^{n}$ is also analytic and bounded by 1 where $\bar{B}_{n}$ is a partial isometry for $n=0,1, \cdots, N$, we have that $\bar{B}_{i} B_{j}=0$ for all $i \neq j$ such that $0 \leqq j \leqq N$. Therefore for $k=1,2, \cdots, N+1, F_{k}(z)=0$ in the above identity, and the $n$th coefficient of $G_{k}(z)$ becomes

$$
-\sum_{j=0}^{n} \bar{B}_{j} f_{k-n-1+j}(0)+\sum_{j=0}^{n} \bar{B}_{j} B_{j} \bar{B}_{j} f_{k-n-1+j}(0)=0
$$

for $n=0,1, \cdots, k-1$, since $\bar{B}_{j}$ is a partial isometry for $j=0,1, \cdots, N$. Hence, $D^{k} D^{* k} D^{k}=D^{k}$ for $k=1,2, \cdots, N+1$.

Conversely, suppose that $D$ is a partial isometry on $\mathscr{D}(B)$. Therefore, $G_{1}(z)=0=-\bar{B}_{0} f(0)+B^{*}(z) B_{0} \bar{B}_{0} f(0)$ for every $f(z)$ in $\mathscr{H}(B)$. If $c$ is any vector orthogonal to $f(0)$ for every $f(z)$ in $\mathscr{H}(B)$, then $K(0, z) c=0$. It follows that $\bar{B}_{0}=\bar{B}_{0} B_{0} \bar{B}_{0}$.

Suppose that $D, D^{2}, \cdots, D^{N+1}$ are partial isometries on $\mathscr{D}(B)$. By induction, we may assume that $B_{0}, B_{1}, \cdots, B_{N-1}$ are partial isometries on $\mathscr{C}$ and consequently $B_{i} \bar{B}_{j}=\bar{B}_{i} B_{l}=0$ for all $i \neq j$ where either $0 \leqq i \leqq N-1$ or $0 \leqq j \leqq N-1$. By the above identity, $G_{N+1}(z)=0$, and therefore the coefficient of $z^{N}$ in the expansion of $G_{N+1}(z)$ vanishes, i.e.,

$$
0=-\sum_{j=0}^{N} \bar{B}_{j} f_{j}(0)+\sum_{j=0}^{N} \bar{B}_{J} B_{J} \bar{B}_{j} f_{j}(0)=-\bar{B}_{N} f_{N}(0)+\bar{B}_{N} B_{N} \bar{B}_{N} f_{N}(0)
$$

for every $f(z)$ in $\mathscr{H}(B)$. If $c$ is any vector orthogonal to $f_{N}(0)$ for every $f(z)$ in $\mathscr{H}(B)$, then

$$
R(0)^{* N} K(0, z) c=0=z \cdot R(0)^{* N-1} K(0, z) c-B(z) \bar{B}_{N} c
$$

and consequently $c-\sum_{j=0}^{N} B_{j} \bar{B}_{j} c=0$. It follows that $\bar{B}_{N}=\bar{B}_{N} B_{N} \bar{B}_{N}$.

THEOREM 2.1. Let $\mathscr{H}(B)$ in $\mathscr{H}^{2}(\mathscr{C})$ be a de Branges-Rovnyak space with reproducing kernel function $K(w, z)$. Every subspace of $\mathscr{H}(B)$ of the form

$$
\underset{B}{\vee}\left\{R(0)^{* n} K(0, z) S: n=0,1,2, \cdots\right\}
$$


where $S$ is a subspace of $\mathscr{C}$ which is invariant under $A_{i j}=B_{l} \bar{B}_{I}$ for all $i, j=0,1,2, \cdots$, is reducing for the backward shift $R(0)$ on $\mathscr{H}(B)$. Conversely, if either $B(0)$ is a partial isometry on $\mathscr{C}$ or the difference quotient identity holds in $\mathscr{H}(B)$, then these subspaces are the only reducing subspaces of $R(0)$ on $\mathscr{H}(B)$.

Proof. The subspaces of the given form reduce $R(0)$ by Lemma 2.1.

Conversely, let $M$ reduce $R(0)$ on $\mathscr{H}(B)$ where $B(0)$ is a partial isometry on $\mathscr{C}$. By Lemma $2.2, D$ is a partial isometry on $\mathscr{D}(B)$. For an arbitrary element $(f(z), g(z))$ in $\mathscr{D}(B)$, if $(a(z), b(z))$ is the projection of $(f(z), g(z))$ onto the kernel of $D$, then $a(z)=a(0)$ and $b(z)=$ $\left(B^{*}(z)-B^{*}(0)\right) / z a(0)$; and if $(c(z), d(z))$ is the projection of $(f(z), g(z))$ onto the range of $D^{*}$ then $c(0)=0$. Therefore the kernel of $D$ is the set of vectors of the form $\left(f(0),\left(\left(B^{*}(z)-B^{*}(0)\right) / z\right) f(0)\right)$ for $f(z)$ in $\mathscr{H}(B)$. It follows that $f(0)$ is in $\mathscr{H}(B)$ and $\|f(0)\|_{B}=\|f(0)\|$ for every $f(z)$ in $\mathscr{H}(B)$.

Let $f(z)$ be in $M$. If $f(0)=g(z)+h(z)$ with $g(z)$ in $M$ and $h(z)$ orthogonal to $M$, then $0=R(0) g(z)+R(0) h(z)$ and consequently $h(z)=h(0)$ since $M$ reduces $R(0)$. Therefore,

$$
\begin{aligned}
\|h(z)\|_{B}^{2} & =\langle h(0), f(0)-g(z)\rangle_{B}=\langle h(0), f(0)-g(0)\rangle \\
& =\langle h(z), f(z)-g(z)\rangle_{B}=0,
\end{aligned}
$$

and $f(0)=g^{\prime}(z)$ is in $M$.

Let $S=\{f(0): f(z) \in M\}$. Then $M=\vee_{B}\left\{R(0)^{* n} K(0, z) S: \quad n=\right.$ $0,1,2, \cdots\}$ and since $R(0)^{* n} K(0, z) S=z R(0)^{* n-1} K(0, z) S-B(z) \bar{B}_{n} S$ for $n \geqq 1$, it follows that $B_{i} \bar{B}_{l} S \subseteq S$ for every $i, j=0,1,2, \cdots$.

Next, suppose that $M$ reduces $R(0)$ on $\mathscr{H}(B)$ and that the difference quotient identity holds. Let $N=\vee\left\{T_{z}^{n} M: n=0,1,2, \cdots\right\}$. Clearly $N$ reduces $T_{z}$ on $\mathscr{H}^{2}(\mathscr{C})$. To show $N$ reduces $1-T_{B} T_{B}^{*}$, we first prove that $M=C L_{B}\left(1-T_{B} T_{B}^{*}\right) M$. But since $\Sigma_{n \geqq 0} R(0)^{* n}\left(1-R(0)^{*} R(0)\right) R(0)^{n}$ converges weakly to $1-T_{B} T_{B}^{*}$ in $\mathscr{H}(B)$ and $M$ is closed under weak limits, we have that $C L_{B}\left(1-T_{B} T_{B}^{*}\right) M \subseteq M$. It follows from [1, Theorem 4] that $C L_{B}\left(1-T_{B} T_{B}^{*}\right) M=M$.

Since $M \subset N$, we have that

$$
C L_{B}\left(1-T_{B} T_{B}^{*}\right) M=M \subset C L_{B}\left(1-T_{B} T_{B}^{*}\right) N .
$$

Let $f(z)$ be in $\mathscr{H}(B) \ominus M$. Then $f(z)$ is in $\mathscr{H}(B) \ominus C L_{B}\left(1-T_{B} T_{B}^{*}\right) N$ since for any $g(z)$ in $M$ and for any nonnegative integer $n$,

$$
\begin{aligned}
\left\langle f(z),\left(1-T_{B} T_{B}^{*}\right) T_{z}^{n} g(z)\right\rangle_{B} & =\left\langle f(z), T_{z}^{n} g(z)\right\rangle \\
& =\left\langle R(0)^{n} f(z),\left(1-T_{B} T_{B}^{*}\right) g(z)\right\rangle_{B} \\
& =0 .
\end{aligned}
$$


Since $f(z)$ was arbitrary, we therefore conclude that $M=$ $C L_{B}\left(1-T_{B} T_{B}^{*}\right) N \subseteq N$, and the desired form of $M$ now follows from Lemma 2.1 .

When the dimension of the coefficient space $\mathscr{C}$ is one and $B(z)$ is not a constant, the range of $1-R(0) R(0)^{*}$ is one-dimensional and therefore the only possible nontrival reducing subspaces of $R(0)$ on $\mathscr{H}(B)$ are the closed span of the polynomials in $\mathscr{H}(B)$ and its orthogonal complement (necessarily, the polynomials must be orthogonal to $(B(z)-B(0)) / z$ in $\mathscr{H}(B))$. However, according to Theorem 2.1 , these possibilities fail to exist under any of the following conditions on $B(z)$.

Corollary 2.1. Let $\mathscr{H}(B)$ be a de Branges-Rovnyak space and suppose the dimension of the coefficient space is one. If either $B(z)$ is a polynomial, $B(0)=0$, or the difference quotient identity holds in $\mathscr{H}(B)$, then $R(0)$ on $\mathscr{H}(B)$ has no nontrivial reducing subspaces.

We recall that if $T$ is a contraction on a Hilbert space $\mathscr{H}$, then there exists a unique minimal co-isometry $V$ acting on a Hilbert space $\mathscr{K}$ containing $\mathscr{H}$ such that $T$ is the restriction of $V$ to $\mathscr{H}[\mathbf{1 1}$, Theorem 4.1]. The space $\mathscr{H}$ is said to be hyperinvariant for $V$ if $\mathscr{H}$ is invariant for every operator that commutes with $V$. The contraction $T$ belongs to the class $C_{0}$. If $\lim _{n \rightarrow \infty}\left\|T^{n} f\right\|=0$ for every vector $f$ of $\mathscr{H}[11]$.

THEOREM 2.2. Suppose that $T$ is a $C_{0}$-contraction on a Hilbert space $\mathscr{H}$, and that $\mathscr{H}$ is hyperinvariant for the minimal co-isometric extension of $T$. Then the reducing subspaces of $T$ are exactly of the form $M=\vee\left\{T^{* n} S\right.$ : $n=0,1,2, \cdots\}$ where $S$ is an arbitrary subspace of $\mathscr{C}$, the closure of the range of $1-T^{*} T$. In this case, $H \Theta M=\vee\left\{T^{* n}(\mathscr{C} \ominus S): n=0,1,2, \cdots\right\}$.

Proof. By the canonical model of de Branges and Rovnyak, $T$ is unitarily equivalent to the backward shift $R(0)$ on $\mathscr{H}(B)$ where $B(z)$ is an inner function and the space $\mathscr{H}(B)$ is hyperinvariant for $T_{z}^{*}$ on $\mathscr{H}^{2}(\mathscr{C})$. Hence by $[11$, p. 199] and [4, Theorem 5], $B(z)$ is a scalar inner function. Therefore, since $A_{i j}=B_{i} \bar{B}_{j}$ is a scalar multiple of the identity for all $i, j=0,1,2, \cdots$, Theorem 2.2 follows from Theorem 2.1.

Theorem 2.1 has a direct counterpart for an arbitrary $C_{0}$-contraction in terms of its minimal co-isometric extension. The proof of Theorem 2.1 may be modified to verify the following.

THEOREM 2.3. Suppose that $T$ is a $C_{0}$-contraction on a Hilbert space $\mathscr{H}$ with minimal co-isometric extension $V$ on $\mathscr{K}$ containing $\mathscr{H} . \quad$ Let $P_{\mathscr{H}}$ be the orthogonal projection of $\mathscr{K}$ onto $\mathscr{H}$, and let $\mathscr{C}$ be the kernel of $V$. The following are equivalent for a subspace $M$ of $\mathscr{H}$. 
(1) $M$ reduces $T$ on $\mathscr{H}$.

(2) $M=\mathscr{H} \cap N$, for some subspace $N$ of $\mathscr{K}$ which reduces both $V$ and $P_{\mathscr{H}}$ on $\mathscr{K}$.

(3) $\quad M=\vee\left\{T^{* n} P_{\mathscr{H}}\left(1-V^{*} V\right) P_{\mathscr{H}} S: n=0,1,2, \cdots\right\}$, for some unique subspace $S$ of $\mathscr{C}$ which is invariant under $A_{i j}=\left(1-V^{*} V\right) V^{i} P_{\mathscr{H}} V^{* j} P_{\mathscr{K}}$ for all $i, j=0,1,2, \cdots$.

In this case,

$$
\begin{aligned}
\mathscr{H} \ominus M & =\mathscr{H} \cap(\mathscr{K} \ominus N) \\
& =\vee\left\{T^{* n} P_{\mathscr{H}}\left(1-V^{*} V\right) P_{\mathscr{H}}(\mathscr{C} \Theta S): n=0,1,2, \cdots\right\} .
\end{aligned}
$$

3. Power partial isometries. We recall that an operator $T$ on Hilbert space is a power partial isometry if $T^{k}$ is a partial isometry for every $k=1,2, \cdots$. P. R. Halmos and L. J. Wallen in [10] characterized in a unique manner an arbitrary power partial isometry as a direct sum of unitary operators, pure isometries, pure co-isometries, and truncated shifts, with each type of summand occurring at most once. The following result enables us to generalize this structure to partial isometries $T$ with no isometric part such that $T^{k}$ is a partial isometry for every $k=$ $1,2, \cdots, N+1$.

THEOREM 3.1. Let $B(z)=\Sigma B_{n} z^{n}$ be analytic and bounded by 1 where $B_{n}$ is a partial isometry on the coefficient space $\mathscr{C}$ for $n=0,1, \cdots, N$. Let $\mathscr{C}_{k}$ be the range of $B_{k}$ for $k=1,2, \cdots, N$ and let $\mathscr{C}_{N+1}=\bigcap_{k=0}^{N} \operatorname{ker} \bar{B}_{k}$. A subspace $M$ of $\mathscr{H}(B)$ reduces $R(0)$ on $\mathscr{H}(B)$ if and only if

$$
\begin{aligned}
M= & \vee\left\{\left(\sum_{k=n+1}^{N+1} \oplus S_{k}\right) z^{n}: n=0,1, \cdots, N\right\} \\
& \bigoplus \underset{B}{\vee}\left\{R(0)^{* n} S_{N+1}: n=N+1, N+2, \cdots\right\}
\end{aligned}
$$

for some subspace $S_{k}$ of $\mathscr{C}_{k}$ for $k=1,2, \cdots, N+1$ such that $B_{j} \bar{B}_{j} S_{N+1} \subseteq S_{N+1}$ for all $i, j=N+1, N+2, \cdots$. In this case,

$$
\begin{aligned}
\mathscr{H}(B) \ominus M= & \vee\left\{\left(\sum_{k=n+1}^{N+1} \oplus\left(\mathscr{C}_{k} \ominus S_{k}\right)\right) z^{n}: n=0,1, \cdots, N\right\} \\
& \oplus \underset{B}{\vee}\left\{R(0)^{* n}\left(\mathscr{C}_{N+1} \ominus S_{N+1}\right): n=N+1, N+2, \cdots\right\} .
\end{aligned}
$$

Proof. Suppose that $M$ reduces $R(0)$ on $\mathscr{H}(B)$. By Lemma 2.2 and Theorem 2.1, a direct computation shows that 


$$
\begin{aligned}
M= & \vee\left\{\left[\prod_{k=0}^{n}\left(1-B_{k} \bar{B}_{k}\right) S\right] z^{n}: n=0,1, \cdots, N\right\} \\
& \oplus \underset{B}{\vee}\left\{R(0)^{* n} K(0, z) S: n=N+1, N+2, \cdots\right\}
\end{aligned}
$$

where $S$ is a subspace of $\mathscr{C}$ which is invariant under $A_{i j}=B_{i} \bar{B}_{,}$for all $i, j=N+1, N+2, \cdots$ and under $A_{i i}=B_{i} \bar{B}_{i}$ for all $i=0,1, \cdots, N$. Since $S=B_{k} \bar{B}_{k} S \oplus\left(1-B_{k} \bar{B}_{k}\right) S$ for $k=0,1, \cdots, N$, we have that $S=$ $\Pi_{0}^{N}\left(1-B_{k} \bar{B}_{k}\right) S \oplus \sum_{k=0}^{N} \oplus B_{k} \bar{B}_{k} S$. Therefore, $\Pi_{0}^{n}\left(1-B_{k} \bar{B}_{k}\right) S=\sum_{k=n+1}^{N+1} \oplus S_{k}$ for $n=0,1, \cdots, N$, and $R(0)^{* n} K(0, z) S=R(0)^{* n} S_{N+1}$ for $n=$ $N+1, N+2, \cdots$, where $S_{k} \subseteq \mathscr{C}_{k}$ for $k=1,2, \cdots, N+1$ and $B_{l} \bar{B}_{,} S_{N+1} \subseteq$ $S_{N+1}$ for all $i, j=N+1, N+2, \cdots$.

Since $\mathscr{C} \ominus S=B_{k} \bar{B}_{k}(\mathscr{C} \ominus S) \oplus\left(1-B_{k} \bar{B}_{k}\right)(\mathscr{C} \ominus S)$ and $B_{k} \bar{B}_{k} \mathscr{C}=$ $B_{k} \bar{B}_{k} S \oplus B_{k} \bar{B}_{k}(\mathscr{C} \ominus S)$ for $k=0,1, \cdots, N$, and

$$
\begin{aligned}
\mathscr{C}_{N+1} & =\prod_{0}^{N}\left(1-B_{k} \bar{B}_{k}\right) \mathscr{C}=\prod_{0}^{N}\left(1-B_{k} \bar{B}_{k}\right) S \oplus \prod_{0}^{N}\left(1-B_{k} \bar{B}_{k}\right)(\mathscr{C} \ominus S) \\
& =S_{N+1} \oplus\left(\mathscr{C}_{N+1} \ominus S_{N+1}\right),
\end{aligned}
$$

the form of $\mathscr{H}(B) \ominus M$ is obtained similarly.

Conversely, if $M$ is of the above form, let $S=\sum_{k=1}^{N+1} \oplus S_{k}$. Then $B_{i} \bar{B}_{j} S \subseteq S$ for every $i, j=0,1,2, \cdots$, and

$$
\begin{aligned}
M= & \vee\left\{\left[\prod_{k=0}^{n}\left(1-B_{k} \bar{B}_{k}\right) S\right] z^{n}: n=0,1, \cdots, N\right\} . \\
& \oplus \underset{B}{\vee}\left\{R(0)^{* n} K(0, z) S: n=N+1, N+2, \cdots\right\} \\
= & \vee_{B}\left\{R(0)^{* n} K(0, z) S: n=0,1,2, \cdots\right\} .
\end{aligned}
$$

Therefore $M$ reduces $R(0)$ by Theorem 2.1 .

The operator $R(0)$ on the following $\mathscr{H}(B)$-space is the canonical model for an arbitrary power partial isometry with no isometric part.

CoROllaRY 3.1. Let $B(z)=\Sigma B_{n} z^{n}$ formally where $\left\{B_{n}: n=\right.$ $0,1,2, \cdots\}$ is a sequence of partial isometries on the Hilbert space $\mathscr{C}$ such that $B_{i} \bar{B}_{j}=\bar{B}_{i} B_{j}=0$ for all $i$ and $j$ such that $i \neq j . \quad$ Let $\mathscr{C}_{k}$ be the range of $B_{k}$ for $k=0,1,2, \cdots$, and let $\mathscr{C}_{\infty}=\bigcap_{k \geqq 0} \operatorname{ker} \bar{B}_{k}$. Then $B(z)$ defines an analytic inner function, and a subspace $M$ of $\mathscr{H}(B)$ reduces $R(0)$ on $\mathscr{H}(B)$ if and only if 


$$
M=\Sigma \oplus\left(S_{\infty} \oplus \sum_{k \geqq n+1} \oplus S_{k}\right) z^{n}
$$

for some subspace $S_{k}$ of $\mathscr{C}_{k}$ for $k=0,1,2, \cdots, \infty$. In this case,

$$
\mathscr{H}(B) \ominus M=\Sigma \oplus\left[\left(\mathscr{C}_{\infty} \ominus S_{\infty}\right) \oplus \sum_{k \geqq n+1} \oplus\left(\mathscr{C}_{k} \ominus S_{k}\right)\right] z^{n}
$$

Proof. Let $c$ be in $\mathscr{C}$ and let $c_{n}$ be the orthogonal projection of $c$ onto the initial space of $B_{n}$ for $n=0,1,2, \cdots$. Then for $|w|<1$,

$$
|B(w) c|^{2}=\Sigma\left|B_{n} c\right|^{2}|w|^{2 n}=\Sigma\left|c_{n}\right|^{2}|w|^{2 n} \leqq \Sigma\left|c_{n}\right|^{2} \leqq|c|^{2} .
$$

Therefore $B(z)$ is analytic and $|B(w)| \leqq 1$ for each $|w|<1$. The reducing subspace structure of $R(0)$ on $\mathscr{H}(B)$ follows from Theorem 3.1. In particular, $\mathscr{H}(B)$ is contained in $\mathscr{H}^{2}(\mathscr{C})$ isometrically so that $B(z)$ is inner.

THEOREM 3.2. Let T be a partial isometry on a Hilbert space $\mathscr{H}$ such that $T^{2}, T^{3}, \cdots, T^{N+1}$ are partial isometries, and suppose that $T$ has no isometric part. Let

$$
\mathscr{C}_{k}=\left(\operatorname{ker} T \cap \operatorname{ker} T^{* k}\right) \ominus\left(\operatorname{ker} T \cap \operatorname{ker} T^{* k-1}\right) \quad \text { for } \quad k=1,2, \cdots, N \text {, }
$$

and let $\mathscr{C}_{N+1}=\bigcap_{k=1}^{N}\left(\operatorname{ker} T \cap \operatorname{ran} T^{k}\right)$. A subspace $M$ of $\mathscr{H}$ reduces $T$ if and only if

$$
M=\sum_{n=0}^{N} \oplus T^{* n}\left(\sum_{k=n+1}^{N+1} \oplus S_{k}\right) \oplus \vee\left\{T^{* n} S_{N+1}: n=N+1, N+2, \cdots\right\}
$$

for some subspace $S_{k}$ of $\mathscr{C}_{k}$ for $k=1,2, \cdots, N+1$, such that $S_{N+1}$ is invariant under $\left(1-T^{*} T\right) T^{i} T^{* \prime}$ for all $i, j=N+1, N+2, \cdots$. In this case,

$$
\begin{aligned}
\mathscr{H} \ominus M= & \sum_{n=0}^{N} \oplus T^{* n}\left(\sum_{k=n+1}^{N+1} \oplus\left(\mathscr{C}_{k} \ominus S_{k}\right)\right) \\
& \oplus \vee\left\{T^{* n}\left(\mathscr{C}_{N+1} \ominus S_{N+1}\right): n=N+1, N+2, \cdots\right\} .
\end{aligned}
$$

Proof. By the canonical model of de Branges and Rovnyak, $T$ is unitarily equivalent to $R(0)$ on $\mathscr{H}(B)$ in which the difference quotient identity holds. Since

$$
\left\langle\left(1-R(0)^{*} R(0)\right) f(z), f(z)\right\rangle_{B}=\langle K(0, z) f(0), f(z)\rangle_{B}
$$

for every $f(z)$ in $\mathscr{H}(B)$, it follows by polarization that (1$\left.R(0)^{*} R(0)\right) f(z)=K(0, z) f(0)$ for every $f(z)$ in $\mathscr{H}(B)$. Since 


$$
1-R(0)^{* k} R(0)^{k}=\sum_{n=0}^{k-1} R(0)^{* n}\left(1-R(0)^{*} R(0)\right) R(0)^{n}
$$

and $1-R(0)^{* k} R(0)^{k}$ is the projection onto the kernel of $R(0)^{k}$ for $k=1,2, \cdots, N+1$, it follows by induction that . $\bar{B}_{n} f_{n}(0)=0$ $(n=0,1, \cdots, N)$ for every $f(z)=\Sigma f_{n}(0) z^{n}$ in $\mathscr{H}(B)$. Hence if $D$ is the difference quotient operator on $\mathscr{D}(B)$, then $\left\|D^{* k} D^{k}(f(z), g(z))\right\|_{\mathscr{D}}=$ $\left\|D^{k}(f(z), g(z))\right\|_{\mathscr{D}}(k=1,2, \cdots, N+1)$ for every $(f(z), g(z))$ in $\mathscr{D}(B)$. Therefore by Lemma $2.2, B_{0}, B_{1}, \cdots, B_{N}$ are partial isometries on the coefficient space $\mathscr{C}$. The structure of the reducing subspaces of $T$ now follows from Theorem 3.1 and the following identities where $f(z)$ is an arbitrary element of $\mathscr{H}(B)$ and $A_{i j}=B_{i} \bar{B}_{j}$ :

$$
\begin{aligned}
& \left(1-R(0)^{*} R(0)\right) K(0, z) f(0)=K(0, z) f(0)-K(0, z) A_{00} f(0) \\
& \left(1-R(0)^{*} R(0)\right) R(0)^{i} K(0, z) f(0)=K(0, z) A_{i 0} f(0)
\end{aligned}
$$

for $i=1,2, \cdots$

$$
\left(1-R(0)^{*} R(0)\right) R(0)^{* j} K(0, z) f(0)=K(0, z) A_{0_{J}} f(0)
$$

for $j=1,2, \cdots$

$$
\begin{gathered}
\left(1-R(0)^{*} R(0)\right) R(0)^{i-1}\left(1-R(0) R(0)^{*}\right) R(0)^{* j-1} K(0, z) f(0) \\
=K(0, z) A_{i j} f(0) \text { for } i, j=1,2, \cdots
\end{gathered}
$$

In the next section, a direct proof of the above theorem is obtained.

THEOREM 3.3. Let $T$ be a partial isometry on a Hilbert space $\mathscr{H}$ and suppose that $T$ has no isometric part. Then $T^{2}, T^{3}, \cdots, T^{N+1}$ are partial isometries if and only if $T=T_{1} \oplus T_{2} \oplus \cdots \oplus T_{N} \oplus V$ where $T_{j}$ is a truncated shift of index $j$ and $V$ is a partial isometry with no isometric part such that $V V^{*}=I$ on $\vee\left\{V^{* n} \operatorname{ker} V: n=0,1, \cdots, N-1\right\}$. Moreover, the representation so expressed is unique, and every projection $P$ which commutes with $T$ is of the form $P=P_{1} \oplus P_{2} \oplus \cdots \oplus P_{N} \oplus Q$ where $P_{i}$ and $Q$ are projections which commute with $T_{j}$ and $V$ respectively $(j=$ $1,2, \cdots, N)$.

Proof. Necessity follows by rewriting the decomposition of $\mathscr{H}$ in Theorem 3.2 as

$$
\begin{aligned}
\mathscr{H}= & \mathscr{C}_{1} \oplus \vee\left\{\mathscr{C}_{2}, T^{*} \mathscr{C}_{2}\right\} \oplus \cdots \oplus \vee\left\{\mathscr{C}_{N}, T^{*} \mathscr{C}_{N}, \cdots, T^{* N-1} \mathscr{C}_{N}\right\} \\
& \oplus \vee\left\{T^{* n} \mathscr{C}_{N+1}: n=0,1, \cdots\right\} .
\end{aligned}
$$


To prove sufficiency, note that $V^{\prime} V^{* \prime}=I$ on the kernel of $V$ for every $j=1,2, \cdots, N$. Therefore by Theorem 2 in [5], it follows that $V^{j}$ is a partial isometry for every $j=1,2, \cdots, N+1$.

Uniqueness of the above representation and the form of projections commuting with $T$ follow from the explicit nature of the decomposition in Theorem 3.2.

Corollary 3.2 (Halmos-Wallen). An operator $T$ on a Hilbert space $\mathscr{H}$ is a power partial isometry if and only if $T=$ $\left(\Sigma_{1}^{\infty} \oplus T_{1}\right) \oplus U_{+}^{*} \oplus U_{+} \oplus U$ where $T_{1}$ is a truncated shift of index $j$ for every $j=1,2, \cdots, U_{+}$is a unilateral shift, and $U$ is unitary. Moreover, the representation so expressed is unique.

Proof. Suppose that $T^{n}$ is a partial isometry for every $n=1,2, \cdots$. Since

$$
\operatorname{ker}\left(1-T^{* n} T^{n}\right)=\left\{f \in \mathscr{H}:\left\|T^{n} f\right\|=\|f\|\right\}=\mathscr{H} \ominus \operatorname{ker} T^{n}
$$

for $n=1,2, \cdots$, it follows that

$$
\left\{f \in \mathscr{H}: T^{n} f \rightarrow 0(n \rightarrow \infty)\right\}=\vee\left\{\operatorname{ker} T^{n}: n=1,2, \cdots\right\}
$$

and hence that $M_{1}=\left\{f \in \mathscr{H}: T^{n} f \rightarrow 0 \quad(n \rightarrow \infty)\right\}$ reduces $T$. Similarly, $M_{2}=\left\{f \in \mathscr{H}: T^{* n} f \rightarrow 0(n \rightarrow \infty)\right.$ and $\left.\left\|T^{n} f\right\|=\|f\|(n=1,2, \cdots)\right\}$ reduces $T$, and $\mathscr{H}=M_{1} \oplus M_{2} \oplus M_{3}$ where

$$
M_{3}=\left\{f \in \mathscr{H}:\left\|T^{n} f\right\|=\|f\|=\left\|T^{* n} f\right\|(n=1,2, \cdots)\right\} .
$$

Therefore, $\left.T\right|_{M_{1}}$ is a power partial isometry with no isometric part, $\left.T\right|_{M_{2}}$ is a unilateral shift, $M_{3}$ reduces $T$ and $\left.T\right|_{M_{3}}$ is unitary [11, Theorem 3.2]. The form of $T$ now follows from Theorem 3.3.

4. A direct proof of Theorem 3.2. We begin by establishing two auxiliary results, the first of which is similar to [10, Lemma 2].

LEMMA 4.1. If $T$ is a contraction on a Hilbert space $\mathscr{H}$ such that $T^{N+1}$ is a partial isometry for some nonnegative integer $N$, then (1$\left.T^{*} T\right) T^{N} T^{* N}=T^{N} T^{* N}\left(1-T^{*} T\right)$.

Proof. The contraction $E=T^{*} T^{N+1} T^{* N}$ is idempotent and therefore Hermitian.

Lemma 4.2. Suppose that $T, T^{2}, \cdots, T^{N+1}$ are partial isometries on a Hilbert space $\mathscr{H}$. Let 


$$
\mathscr{C}_{k}=\left(\operatorname{ker} T \cap \operatorname{ker} T^{* k}\right) \ominus\left(\operatorname{ker} T \cap \operatorname{ker} T^{* k-1}\right) \quad \text { for } \quad k=1,2, \cdots, N \text {, }
$$

and let $\mathscr{C}_{N+1}=\bigcap_{1}^{N}\left(\operatorname{ker} T \cap \operatorname{ran} T^{k}\right)$. Then $\operatorname{ker} T=\left(\Sigma_{1}^{N} \oplus \mathscr{C}_{k}\right) \oplus \mathscr{C}_{N+1}$, and a subspace $S$ of ker $T$ is invariant under $T^{k} T^{* k}$ for every $k=1,2, \cdots, N$ if and only if $S=\left(\Sigma_{1}^{N} \oplus S_{k}\right) \oplus S_{N+1}$ for some subspace' $S_{k}$ of $\mathscr{C}_{k}$ for $k=1,2, \cdots, N+1$. In this case, $T^{n} T^{* n} S_{k}=S_{k}$ for all $n<k$ $(k=1,2, \cdots, N+1 ; n=1,2, \cdots, N)$.

Proof. By Lemma 4.1, the kernel of $T$ is invariant under $T^{k} T^{* k}$ for all $k=1,2, \cdots, N$. Therefore,

$$
\operatorname{ker} T=\left(\operatorname{ker} T \cap \operatorname{ker} T^{* k}\right) \oplus\left(\operatorname{ker} T \cap \operatorname{ran} T^{k}\right)
$$

for all $k=1,2, \cdots, N$ and consequently $\operatorname{ker} T=\left(\Sigma_{1}^{N} \oplus \mathscr{C}_{k}\right) \oplus \mathscr{C}_{N+1}$.

Suppose $T^{k} T^{* k} S \subseteq S \quad(k=1,2, \cdots, N)$ for some subspace $S$ of ker $T$. Let $S_{k}=\left(S \cap \operatorname{ker} T^{* k}\right) \ominus\left(S \cap \operatorname{ker} T^{* k-1}\right)$ for $k=1,2, \cdots, N$ and let $S_{N+1}=\bigcap_{1}^{N}\left(S \cap \operatorname{ran} T^{k}\right)$. As above, $S=\left(\Sigma_{1}^{N} \oplus S_{k}\right) \oplus S_{N+1}$ and, since $S$ is invariant under $T^{k} T^{* k}$, we have that

\section{$\operatorname{ker} T \cap \operatorname{ker} T^{* k}=\left[S \cap \operatorname{ker} T^{* k}\right] \oplus\left[(\operatorname{ker} T \ominus S) \cap \operatorname{ker} T^{* k}\right]$}

for every $k=1,2, \cdots, N$.

Fix $k(1 \leqq k \leqq N)$ and let $f$ be in $S_{k}$. Then $f$ is in $\operatorname{ker} T \cap \operatorname{ker} T^{* k}$ and $f$ is orthogonal to both $S \cap \operatorname{ker} T^{* k-1}$ and $(\operatorname{ker} T \ominus S) \cap \operatorname{ker} T^{* k-1}$. Therefore $f$ is in $\mathscr{C}_{k}$. Since $f$ and $k$ were arbitrary, it follows that $S_{k} \subseteq \mathscr{C}_{k}$ for every $k=1,2, \cdots, N$. Clearly, $S_{N+1} \subseteq \mathscr{C}_{N+1}$.

Conversely, suppose that $S=\left(\sum_{1}^{N} \oplus S_{k}\right) \oplus S_{N+1}$ for some subspace $S_{k}$ of $\mathscr{C}_{k}(k=1,2, \cdots, N+1)$. Since the kernel of $T$ is invariant under $T^{k} T^{* k}$ we have as above

$$
\operatorname{ker} T^{* k}=\left[\operatorname{ker} T \cap \operatorname{ker} T^{* k}\right] \oplus\left[(\mathscr{H} \ominus \operatorname{ker} T) \cap \operatorname{ker} T^{* k}\right]
$$

for every $k=1,2, \cdots, N$. Therefore $S_{k}$ is contained in the range of $T^{k-1}$ and consequently $S$ is invariant under $T^{k} T^{* k}$ for every $k=1,2, \cdots, N$.

Alternate proof of Theorem 3.2. Let $T$ be a partial isometry with no isometric part such that $T^{2}, T^{3}, \cdots, T^{N+1}$ are partial isometries, and let $M$ reduce $T$. By [6] $M=\vee\left\{T^{* n} S: n=0,1,2, \cdots\right\}$ where $S$ is a subspace of the kernel of $T$ which is invariant under $A_{i j}=\left(1-T^{*} T\right) T^{i} T^{* j}$ for all $i, j=0,1,2, \cdots$. By Lemma 4.1 and $4.2, S=\left(\sum_{1}^{N} \oplus S_{k}\right) \oplus S_{N+1}$ where $S_{k}$ is a subspace of $\mathscr{C}_{k}$ for $k=1,2, \cdots, N+1$ such that $A_{i j} S_{N+1} \subseteq S_{N+1}$ for all $i, j=N+1, N+2, \cdots$, and 


$$
\begin{aligned}
M & =\sum_{n=0}^{N} \oplus T^{* n} S \oplus \vee\left\{T^{* n} S: n=N+1, N+2, \cdots\right\} \\
& =\sum_{n=0}^{N} \oplus T^{* n}\left(\sum_{k=n+1}^{N+1} \oplus S_{k}\right) \oplus \vee\left\{T^{* n} S_{N+1}: n=N+1, N+2, \cdots\right\} .
\end{aligned}
$$

The form of $\mathscr{H} \ominus M$ is obtained similarly since

$$
\text { ker } T \ominus S=\left(\sum_{1}^{N} \oplus\left(\mathscr{C}_{k} \ominus S_{k}\right)\right) \oplus\left(\mathscr{C}_{N+1} \ominus S_{N+1}\right)
$$

and by [6]

$$
\mathscr{H} \ominus M=\vee\left\{T^{* n}(\operatorname{ker} T \ominus S): n=0,1,2, \cdots\right\} .
$$

Conversely, if $M$ is of the above form, let $S=\left(\sum_{1}^{N} \oplus S_{k}\right) \oplus S_{N+1}$. By Lemmas 4.1 and 4.2, $S$ is invariant under $\left(1-T^{*} T\right) T^{i} T^{* j}$ for every $i, j=0,1,2, \cdots$, and $M=\vee\left\{T^{* n} S: n=0,1,2, \cdots\right\}$. Therefore by [6] $M$ reduces $T$.

ACKNOWLedgements. I am grateful to James A. Deddens for some helpful conversations concerning this paper. I also wish to thank the referee for suggesting some of the generalizations included.

\section{REFERENCES}

1. L. de Branges and J. Rovnyak, Canonical models in quantum scattering theory, Perturbation Theory and its Applications in Quantum Mechanics (Proc. Adv. Sem. Math. Res. Center, U.S. Army, Theoret. Chem. Inst., Univ. of Wisconsin, Madison, Wis., 1965), Wiley, New York, 1966, pp. 295-392.

2. - Square Summable Power Series, Holt, Rinehart and Winston, New York, 1966.

3. A. Brown, On a class of operators, Proc. Amer. Math. Soc., 4 (1953), 723-728.

4. R. G. Douglas and C. Pearcy, On a topology for invariant subspaces, J. Functional Anal., 2 (1968), 323-341.

5. I. Erdelyi, Partial isometries closed under multiplication on Hilbert spaces, J. Math. Anal. Appl., 22 (1968), 546-551.

6. J. Guyker, Reducing subspaces of contractions with no isometric part, Proc. Amer. Math. Soc., 3 (1974), 411-413.

7. - Reducing subspaces of contractions with no isometric part, Thesis, Lehigh University, Bethlehem, Pa., 1970.

8. P. R. Halmos, A Hilbert Space Problem Book, Van Nostrand, Princeton, N.J., 1967.

9. - Shifts on Hilbert spaces, J. Reine Angew. Math., 208 (1961), 102-112.

10. P. R. Halmos and L. J. Wallen, Powers of partial isometries, J. Math. Mech., 19(1970), 657-663.

11. B. Sz.-Nagy and C. Foiaş, Analyse harmonique des opérateurs de l'espace de Hilbert, Masson, Paris; Akad. Kiadó, Budapest, 1967; English rev. Transl., North-Holland, Amsterdam; American Elsevier, New York; Akad. Kiadó, Budapest, 1970.

Received March 12, 1975 and in revised form January 20, 1976. This research was partially supported by a SUNY Summer Research Fellowship. 



\section{PACIFIC JOURNAL OF MATHEMATICS}

\section{EDITORS}

RICHARD ARENS (Managing Editor)

University of California

Los Angeles, California 90024

\section{J. Dugundu}

Department of Mathematics University of Southern California Los Angeles, California 90007

D. Gilbarg and J. Milgram Stanford University Stanford, California 94305

\section{ASSOCIATE EDITORS}
E. F. BECKENBACH
B. H. NeumanN
F. WOLF
K. YoSHIDA

\section{SUPPORTING INSTITUTIONS}

UNIVERSITY OF BRITISH COLUMBIA CALIFORNIA INSTITUTE OF TECHNOLOGY

UNIVERSITY OF CALIFORNIA

MONTANA STATE UNIVERSITY

UNIVERSITY OF NEVADA

NEW MEXICO STATE UNIVERSITY

OREGON STATE UNIVERSITY

UNIVERSITY OF OREGON

OSAKA UNIVERSITY

\author{
UNIVERSITY OF SOUTHERN CALIFORNIA \\ STANFORD UNIVERSITY \\ UNIVERSITY OF HAWAII \\ UNIVERSITY OF TOKYO \\ UNIVERSITY OF UTAH \\ WASHINGTON STATE UNIVERSITY \\ UNIVERSITY OF WASHINGTON \\ AMERICAN MATHEMATICAL SOCIETY
}

The Supporting Institutions listed above contribute to the cost of publication of this Journal, but they are not owners or publishers and have no responsibility for its contents or policies.

Mathematical papers intended for publication in the Pacific Journal of Mathematics should be in typed form or offset-reproduced (not dittoed), double spaced with large margins. Underline Greek letters in red, German in green, and script in blue. The first paragraph or two must be capable of being used separately as a synopsis of the entire paper. Items of the bibliography should not be cited there unless absolutely necessary, in which case they must be identified by author and Journal, rather than by item number. Manuscripts, in duplicate, may be sent to any one of the four editors. Please classify according to the scheme of Math. Reviews, Index to Vol. 39. All other communications should be addressed to the managing editor, or Elaine Barth, University of California, Los Angeles, California, 90024.

100 reprints are provided free for each article, only if page charges have been substantially paid. Additional copies may be obtained at cost in multiples of 50 .

The Pacific Journal of Mathematics is issued monthly as of January 1966. Regular subscription rate: $\$ 72.00$ a year (6 Vols., 12 issues). Special rate: $\$ 36.00$ a year to individual members of supporting institutions.

Subscriptions, orders for back numbers, and changes of address should be sent to Pacific Journal of Mathematics, 103 Highland Boulevard, Berkeley, California, 94708.

PUBLISHED BY PACIFIC JOURNAL OF MATHEMATICS, A NON-PROFIT CORPORATION

Printed at Jerusalem Academic Press, POB 2390, Jerusalem, Israel.

$$
\begin{gathered}
\text { Copyright } 1976 \text { Pacific Journal of Mathematics } \\
\text { All Rights Reserved }
\end{gathered}
$$




\section{Pacific Journal of Mathematics}

Vol. 62, No. 2

February, 1976

Allan Russell Adler and Catarina Isabel Kiefe, Pseudofinite fields, procyclic

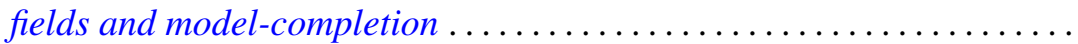

Christopher Allday, The stratification of compact connected Lie group

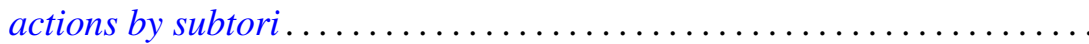

Martin Bartelt, Commutants of multipliers and translation operators .......

Herbert Stanley Bear, Jr., Ordered Gleason parts ..................

James Robert Boone, On irreducible spaces. II .....................

James Robert Boone, On the cardinality relationships between discrete

collections and open covers ............................

L. S. Dube, On finite Hankel transformation of generalized functions .......

Michael Freedman, Uniqueness theorems for taut submanifolds . . . . . . . . .

Shmuel Friedland and Raphael Loewy, Subspaces of symmetric matrices

containing matrices with a multiple first eigenvalue .............

Theodore William Gamelin, Uniform algebras spanned by Hartogs

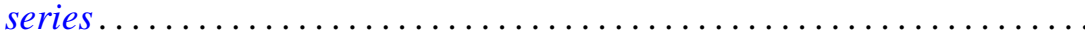

James Guyker, On partial isometries with no isometric part ............

Shigeru Hasegawa and Ryōtarō Satō, A general ratio ergodic theorem for

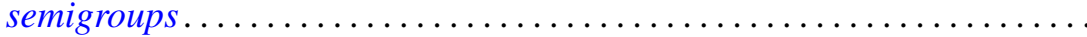

Nigel Kalton and G. V. Wood, Homomorphisms of group algebras with norm less than $\sqrt{2}$.

Thomas Laffey, On the structure of algebraic algebras...

Will Y. K. Lee, On a correctness class of the Bessel type differential operator $S_{\mu}$

Robert D. Little, Complex vector fields and divisible Chern classes ....

Kenneth Louden, Maximal quotient rings of ring extensions . .

Dieter Lutz, Scalar spectral operators, ordered $l^{\rho}$-direct sums, and the

counterexample of Kakutani-McCarthy . .

Ralph Tyrrell Rockafellar and Roger Jean-Baptiste Robert Wets, Stochastic

convex programming: singular multipliers and extended duality

singular multipliers and duality.

Edward Barry Saff and Richard Steven Varga, Geometric overconvergence of rational functions in unbounded domains ..........

Joel Linn Schiff, Isomorphisms between harmonic and P-harmonic Hardy

spaces on Riemann surfaces.

Virinda Mohan Sehgal and S. P. Singh, On a fixed point theorem of

Krasnoselskii for locally convex spaces.

Lewis Shilane, Filtered spaces admitting spectral sequence operations

Michel Smith, Generating large indecomposable continua . 